\title{
Achalasia: what to do in the face of failures of Heller myotomy
}

\author{
Valter N. Felix, ${ }^{1}$ Kenric M. Murayama, ${ }^{2}$ Luigi Bonavina, ${ }^{3}$ iD and Moo In Park ${ }^{4}$ \\ ${ }^{1}$ Nucleus of General and Specialized Surgery - São Paulo and Department of Gastroenterology - Surgical Division - São \\ Paulo University, FMUSP, Sao Paulo, Brazil. 2Department of Surgery, John A. Burns School of Medicine, the University of \\ Hawaii at Manoa, Honolulu, Hawaii. ${ }^{3}$ Division of General and Foregut Surgery, Department of Biomedical Sciences for Health, \\ IRCCS Policlinico San Donato, The University of Milan, San Donato Milanese, Italy. ${ }^{4}$ Department of Internal Medicine, Kosin \\ University College of Medicine, Busan, Korea
}

Address for correspondence: Kenric M. Murayama, MD, MBA, FACS, Department of Surgery, John A. Burns School of Medicine, the University of Hawaii at Manoa, 1356 Lusitana Street, 6th Floor, Honolulu, HI 96813. kenricm@hawaii.edu

Achalasia is a primary motility disorder of the esophagus, and while there are several treatment options, there is no consensus regarding them. When therapeutic intervention for achalasia fails, a careful evaluation of the cause of the persistent or recurrent symptoms using upper endoscopy, esophageal manometry, and contrast radiologic studies is required to understand the cause of therapy failure and guide plans for subsequent treatment. Options for reintervention are the same as for primary intervention and include pneumatic dilation, botulinum toxin injection, peroral endoscopic myotomy, or redo esophageal myotomy. When reintervention fails or if the esophagus is not amenable to intervention and the disease is considered end-stage, esophagectomy is the last option to manage recurrent achalasia.

Keywords: achalasia; therapeutic methods for achalasia; pneumatic dilation; POEM; redo esophageal myotomy; esophagectomy

\section{Introduction}

Achalasia is an unusual disease with an incidence reported at $1 / 100,000$ worldwide. ${ }^{1}$ The disease is characterized by the retention of food in the esophagus owing to poor esophageal transit and emptying with worsening dilation. Achalasia is a progressive disease and treatment is aimed at relieving symptoms by improving esophageal emptying. The longterm outcome data following intervention are very important in deciding which treatment option is optimal for a patient with achalasia. However, few controlled studies on the long-term outcome data following treatment are available, making it difficult for the clinicians recommending treatment. ${ }^{1-3}$

\section{Primary treatment options}

The principle intervention options for achalasia include endoscopically directed botulinum toxin injections (BTIs), endoscopic pneumatic dilation
(PD), surgical esophageal myotomy, and, more recently, peroral endoscopic myotomy (POEM). Treatment selection is dependent on the individual patient's disease and situation and the availability of providers comfortable with each of the treatment options. Importantly, and relevant to this discussion, are the causes of failure of the primary intervention and options for subsequent intervention.

Botulinum toxin injection. Botulinum toxin is a potent inhibitor of acetylcholine (ACh) release into the nerve endings causing short-term muscle paralysis. At the lower esophageal sphincter (LES), BTI interrupts the neurologic component of the smooth muscles causing partial paralysis of the muscles. ${ }^{4}$ Unfortunately, while an appealing therapy owing to a low-risk profile compared with more invasive treatment options, the benefits are generally 
temporary, with most patients requiring additional treatment within 12 months. ${ }^{1}$ In a published Cochrane review, only 29 of 56 BTI patients were improved at 6 months and even fewer (27 of 72 patients) were in remission at 12 months. ${ }^{5}$ Currently, BTI is rarely used as primary therapy and is generally reserved for patients who are not candidates for definitive therapy. ${ }^{1}$

PD. PD has been widely used and is an effective nonsurgical treatment for achalasia. ${ }^{6,7}$ The mechanism of action for PD is the disruption of the muscle fibers of the LES utilizing a pressurized balloon and, often, fluoroscopic guidance. ${ }^{8}$ Progressive dilation is carried out using balloon sizes of 30-, 35-, and 40-mm diameter based on provider comfort level and patient response. ${ }^{8}$ In a recently published metaanalysis of 52 uncontrolled studies, "clinical success, defined by an Eckardt score $\leq 3$, was achieved in $83 \%$ over a follow-up period ranging from 3 to 6 months." ${ }^{\prime}$ Importantly, there was a statistically significant decrease in the average LES pressure after $\mathrm{PD}$ (34.47 versus $20.80 \mathrm{~mm} \mathrm{Hg}, P<$ $0.01) .{ }^{8}$ One difficulty with evaluating the benefit of PD is that studies are inconsistent in the PD treatment algorithm-in particular, there is an inconsistency in the balloon size utilized, the number of repeat interventions, and time between attempted dilations. ${ }^{5}$ The most feared complication of PD is transmural perforation-a systematic review reports contemporary perforation rates $<1 \%$, similar to the unrecognized perforation rate during surgical myotomy ${ }^{1}$; however, many other studies report slightly higher rates of perforation. ${ }^{9}$ Remission rates vary in reports, but in a Cochrane review, 46 of 57 PD patients were in remission at 6 months and 55 of 75 at 12 months. ${ }^{5}$ Importantly, surgical myotomy has a greater success at symptom improvement than single episode PD, but repeated PD may have similar outcomes. ${ }^{10}$

\section{Esophageal myotomy. Esophageal Heller} myotomy (HM) has been an option to treat achalasia for many years, but only after the addition of the minimally invasive approach did laparoscopic HM (LHM) become the gold standard. ${ }^{11}$ The morbidity and mortality after LHM are low, but the patient should be aware of a few potential problems. First is the obvious risk of esophageal perforation while performing the myotomy that extends through the muscle layers to the submucosa and must extend several centimeters up onto the esophagus and down onto the stomach $2-3 \mathrm{~cm}$ to be effective. ${ }^{11}$ Second, the risk of postoperative gastroesophageal reflux disease (GERD) can be substantial and an antireflux operation is recommended to minimize postmyotomy GERD. In general, a partial wrap is selected and a complete $360^{\circ}$ wrap is discouraged because of an increased risk of dysphagia. ${ }^{11}$ Addition of an antireflux procedure significantly reduces GERD symptoms from $31.5 \%$ to $8.8 \%$ with a similar improvement in achalasia symptoms. ${ }^{12}$ Finally, and importantly, patients should be aware of the potential for a recurrence of achalasia symptoms owing to incomplete myotomy, most commonly on its gastric portion; scarring and fibrosis resulting in the loss of LES compliance; and obstruction at the LES owing to the fundoplication. ${ }^{12}$

POEM. POEM is a recent addition to the treatment options for achalasia and involves the endoscopic division of the circular muscle of the esophagus after creating an opening in the esophageal mucosa at some distance proximal to the LES. The muscular division is carried distally onto the stomach side of the LES. ${ }^{13}$ With the disruption of the LES, one concern is the risk of post-POEM GERD. In a recent systematic review, utilizing 24-h $\mathrm{pH}$ monitoring after POEM, the "range of abnormal acid exposure varied from 20 to 53\%; all patients were symptomatically controlled with proton pump inhibitors." 14 In centers with POEM experience, the procedure has been found to be an effective alternative to esophageal myotomy in the treatment of achalasia. ${ }^{14}$

In a multicenter randomized clinical trial that compared POEM with PD as the initial treatment for the treatment of 133 patients with achalasia, 2year treatment success was $92 \%$ in the POEM group versus 54\% in the PD group. ${ }^{15}$ This result indicates a significantly higher treatment success rate at 2 years in POEM compared with PD. However, the incidence of reflux esophagitis was significantly higher in the POEM group than in the PD group (41\% in the POEM group and 7\% in the PD group). In a recent retrospective study, POEM appears to be more effective than PD after 2 years of treatment in patients with all manometric subtypes of achalasia, but the incidence of postprocedure reflux remains 
a concern. ${ }^{16}$ Similarly, a systematic review of postprocedure GERD after POEM compared with LHM reported significantly more reflux symptoms $(19.0 \%$ versus $8.8 \%$ ), abnormal acid exposure (39.0\% versus $16.8 \%)$, and esophagitis (29.4\% versus $7.6 \%)$ after POEM. ${ }^{17}$

\section{Selecting a treatment option}

Although endoscopic BTI is an established treatment modality for achalasia, a meta-analysis comparing BTI with both single PD and HM showed BTI to be less durable than both. ${ }^{18}$ As mentioned earlier, BTI is generally reserved for those achalasia patients who are not candidates for other interventions. As LHM has evolved to be the gold standard intervention to relieve the symptoms and consequences of achalasia, it is important to understand how the other two interventions, PD and POEM, compare (as described above). Although POEM showed a higher success rate after 2 years of treatment than $\mathrm{PD}$, there are no long-term results comparing the two treatments. The International Society for Diseases of the Esophagus published The 2018 ISDE Achalasia Guidelines and recommended that "patients with achalasia who do not respond to initial treatment with graded PD should be recommended for HM or POEM." 19

In 2015, Persson et al. reported the long-term results of the first randomized, controlled, clinical trial comparing the therapeutic outcome of HM with a posterior partial fundoplication with repetitive PD in patients with newly diagnosed idiopathic achalasia. ${ }^{20}$ After 3 and 5 years, the treatment failure rate was significantly lower in the HM group (4\% and $8 \%$, respectively) compared with the PD group ( $8 \%$ and $36 \%$, respectively). ${ }^{20}$

In 2019, Harvey et al. reported that durability of up to three PDs and surgical HM over 10 years of a follow-up was $86.2 \%$ and $81.9 \%$, respectively, in a large national database study involving 6938 subjects. ${ }^{21}$ Long-term treatment effects of PD over 10 years seem better than HM in this largest study of the outcomes of achalasia therapy, but perforation occurred in $1.6 \%$ of 4748 patients who underwent PD and in none of 2190 patients who underwent HM. Mortality at 30 days was $1.9 \%$ for PD and there were no deaths in the HM group. ${ }^{21}$

In 2019, Werner et al. reported on a multicenter, randomized trial comparing POEM with LHM +
Dor fundoplication in the treatment of symptomatic achalasia. ${ }^{22}$ At a 2-year follow-up, clinical success was similar in both groups $(83.0 \%$ in the POEM group and $81.7 \%$ in the LHM group) and reflux esophagitis was present in 44\% of POEM patients and $29 \%$ of LHM patients. ${ }^{22}$ The authors concluded that POEM was a noninferior treatment for symptomatic achalasia compared with LHM with Dor fundoplication, but GERD was more common after POEM. $^{22}$

HM has been considered the treatment of choice to treat achalasia with good long-term results, including reflux control; ${ }^{23}$ however, symptoms can persist or recur in $10-20 \%$ of patients. ${ }^{24,25}$ Unfortunately, most reports do not differentiate between the group of patients with persistent versus recurrent symptoms after a period of initial improvement. ${ }^{26}$ Persistence can be defined as an Eckardt score that fails to fall to $\leq 3$ with treatment or as failure to reduce a symptom score by at least $50 \% .{ }^{27}$ Recurrence may be described as an initial successful treatment response followed by an increase in the Eckardt score to $>3 .^{28}$

\section{Causes of persistence or recurrence of dysphagia after surgical myotomy}

A careful evaluation of the cause of the persistent or recurrent symptoms is recommended before a new treatment is conducted. ${ }^{29}$ The most common causes of myotomy failure in the treatment of achalasia include incomplete myotomy, fibrosis, or scarring that decreases compliance at the LES, and obstruction owing to extrinsic compression by the fundoplication.

In patients with persistent dysphagia after surgery, the most common cause is an incomplete myotomy-a failure to carry the myotomy far enough onto the stomach or esophagus. In fact, while exact distances vary in some reports, it is clear that extending the myotomy at least around $6 \mathrm{~cm}$ above and $3 \mathrm{~cm}$ below the gastroesophageal junction (GEJ) is associated with lower rates of postoperative dysphagia requiring reoperation..$^{11,30}$ Scarring is not usually seen in the first 6 months after HM, but even if the Heller procedure was correctly done with an extensive myotomy, the late fibrosis at the site of the myotomy may be a cause of recurrent dysphagia. ${ }^{31}$

Postmyotomy dysphagia can result from twisting or herniation of the fundoplication through the 
diaphragmatic hiatus, but when used after myotomy to prevent reflux, fundoplication has not been a significant cause of dysphagia. When the contrast radiography of the esophagus shows an important angulation in the distal esophagus, suggesting that something is wrong with the previous fundoplication resulting in esophageal obstruction, surgical repositioning of the fundoplication is required. A disrupted or slipped fundoplication after HM, identified by esophagography and endoscopy, may result in reflux and dysphagia. Heartburn, regurgitation, and/or dysphagia after HM with fundoplication can often be treated with postoperative proton pump inhibitors, but when refractory may require revision of the fundoplication. ${ }^{32}$ Esophageal $\mathrm{pH}$ testing, when reflux is suspected, can be helpful in determining the pathophysiology of the recurrence of symptoms. Abrupt drops in $\mathrm{pH}$ characterize postoperative reflux with prolonged exposure of the distal esophagus to refluxate, which may indicate the need for a redo of the fundoplication. ${ }^{29}$

Progression to end-stage achalasia generally follows from the failure of repeated treatment interventions and is characterized by marked dilation, tortuosity, and sigmoidization of the esophagus. ${ }^{33}$ The progression of the disease after HM to endstage achalasia is characterized by the persistence of dysphagia and regurgitation and leaves little alternative to esophagectomy. ${ }^{28,29}$ Among the causes of dysphagia persistence or recurrence in those undergoing myotomy and fundoplication, incomplete myotomy, postoperative fibrosis, and disease progression frequently result in a dilated and tortuous esophagus.

\section{Diagnosis and treatment options for failed myotomy}

Evaluation of patients who have persistent or recurrent symptoms of achalasia after myotomy and fundoplication is similar to the workup before the initial myotomy, but includes several important considerations: knowledge of interventions before the myotomy (as this may affect the selection of subsequent intervention), status of the esophagus and fundoplication anatomy, and assurance that achalasia recurrence or persistence is actually causing the symptoms. A thorough study of such patients is performed with esophageal manometry, upper endoscopy, contrast esophagography, and, sometimes, $\mathrm{pH}$ monitoring. ${ }^{28,34}$
Upper gastrointestinal series (UGI) provides important information about the degree of esophageal dilation, evidence of sigmoidization of the esophagus, the possibility of incomplete myotomy, and the status of fundoplication. Images on UGI after an incomplete myotomy will demonstrate an image that resembles a "bird's beak," similar to the appearance before myotomy. Fibrosis on UGI may appear as an irregular contour of the distal esophagus. ${ }^{35}$

Endoscopy is important in patients who have a recurrence of achalasia symptoms after a successful HM to evaluate mucosal damage and to ensure that there is no cause for secondary achalasia, such as a new malignancy. ${ }^{36}$ In addition to examination of the mucosa under direct visualization, the competency of the LES can be estimated, and retroflexion, once in the stomach, can provide information about possible diaphragmatic hernia.

Physiology studies are important in the further evaluation and planning for subsequent intervention to relieve symptoms of recurrent or persistent achalasia. Esophageal manometry is an important test for confirming the diagnosis of incomplete myotomy (high pressure of LES), fibrosis (low pressure of LES), and maintenance or not of pressurization of the esophageal body. ${ }^{36}$ In a report by Tatum et al., manometry results suggest a modest, but significant, improvement in the esophageal transit of both liquid and viscous boluses after myotomy. ${ }^{37}$ The authors further conclude that there is value in performing manometry and impedance studies in the postintervention follow-up of achalasia patients when symptoms persist or recur. ${ }^{37}$

In the recently published European Guideline on Achalasia - UEG and ESNM recommendations, the authors recommend "treating recurrent or persistent dysphagia after laparoscopic HM with PD, POEM, or redo surgery." 38

\section{Options for the treatment of failed myotomy}

Despite the abundant literature, there is no consensus about the best way to approach the patients with persistence or recurrence of dysphagia after HM owing to incomplete myotomy or local fibrosis: PD, BTI, POEM, redo myotomy, or even esophagectomy, considered the last resort to manage achalasia when all conservative strategies fail or when the disease has reached its end-stage. ${ }^{39}$ 
Table 1. Results of use of PD after failed Heller myotomy

\begin{tabular}{|c|c|c|c|}
\hline Author & Year & $n$ & Success (\%) \\
\hline Guardino et al. ${ }^{40}$ & 2004 & 12 & 50 \\
\hline Zaninotto et al. ${ }^{41}$ & 2008 & 39 & 75 \\
\hline Chen et al. ${ }^{42}$ & 2010 & 11 & 54 \\
\hline Kumbhari et al. ${ }^{43}$ & 2013 & 27 & 96 \\
\hline Legros et al. ${ }^{44}$ & 2014 & 18 & 78 \\
\hline Amani et al. ${ }^{45}$ & 2015 & 30 & 83 \\
\hline Saleh et al. ${ }^{46}$ & 2016 & 21 & 57 \\
\hline Schlottmann et al. ${ }^{47}$ & 2017 & 19 & 63 \\
\hline
\end{tabular}

$n$, number of patients.

\section{Endoscopic procedures}

PD and botulin toxin injection. Retrospective reports, limited in the number of treated patients, report that $\mathrm{PD}$ is effective in relieving achalasia symptoms after failed myotomy in $50-96 \%$ of patients ${ }^{40-47}$ (Table 1). In all studies, during a followup from 3 to 10 years, the achalasia symptom score was assessed at different intervals, and the procedure was repeated in case of symptom relapse (Eckardt $>3$ ). It is not uncommon for each patient to go through more than two PDs to control dysphagia. In the reported experience of Schlottmann et al. (Table 2), another four patients responded positively to PD and BTI, but BTI is infrequently used in the literature to treat recurrent dysphagia after myotomy. ${ }^{47}$

Comparing patients treated with PD or revisional surgery after failed myotomy, Vela et al. reported similarity of results regarding symptom control and improvement in radiological esophageal emptying time. ${ }^{34}$ This may support a preference for treating the recurrence of dysphagia after myotomy with PD

Table 2. Results of POEM after failed Heller myotomy

\begin{tabular}{|c|c|c|c|}
\hline Author & Year & $n$ & Success (\%) \\
\hline Onimaru et al. ${ }^{49}$ & 2013 & 10 & 100 \\
\hline Zhou et al. ${ }^{50}$ & 2013 & 12 & 91.7 \\
\hline Vigneswaran et al. ${ }^{51}$ & 2014 & 5 & 100 \\
\hline Orenstein et al. ${ }^{48}$ & 2015 & 3 & 100 \\
\hline Fumagalli et al. ${ }^{52}$ & 2016 & 6 & 100 \\
\hline Ngamruengphong et al. ${ }^{54}$ & 2017 & 90 & 81 \\
\hline Zhang et al..$^{53}$ & 2018 & 46 & 95.7 \\
\hline
\end{tabular}

$n$, number of patients.
Table 3. Results of redo-myotomy and fundoplication

\begin{tabular}{|c|c|c|c|}
\hline Author & Year & $n$ & Success (\%) \\
\hline Serra et al. ${ }^{31}$ & 1998 & 14 & 100 \\
\hline Rosati et al. ${ }^{55}$ & 1998 & 15 & 93 \\
\hline Gorecki et al. ${ }^{56}$ & 2002 & 8 & 87.5 \\
\hline Duffy et al. ${ }^{57}$ & 2003 & 5 & 100 \\
\hline Iqbal et al. ${ }^{58}$ & 2006 & 15 & 80 \\
\hline Grotenhuis et al. ${ }^{59}$ & 2007 & 12 & 89 \\
\hline Rakita et al. ${ }^{60}$ & 2007 & 12 & 73 \\
\hline Herbella et al. ${ }^{61}$ & 2008 & 25 & 88 \\
\hline Patti and Allaix ${ }^{29}$ & 2015 & 40 & 79 \\
\hline Fumagalli et al. ${ }^{52}$ & 2016 & 9 & 100 \\
\hline Veenstra et al. ${ }^{62}$ & 2016 & 58 & 64 \\
\hline Smith et al. ${ }^{63}$ & 2019 & 34 & 69 \\
\hline
\end{tabular}

$n$, number of patients.

before proposing an invasive procedure, although multiple dilations may be required.

POEM. Patients having failure of HM often still have an intact antireflux procedure resulting in an ideal scenario for the application of POEM. When performing the endoscopic myotomy, the proceduralist must avoid the previous surgical myotomy, which is commonly carried out on the anterior esophageal wall. The procedure is facilitated by placing the patient in the lateral decubitus position and performing myotomy at the $2 \mathrm{o}^{\prime}$ clock position. ${ }^{48}$ Many small series show a $91-100 \%$ effectiveness of POEM in patients with surgical myotomy failure ${ }^{48-53}$ and the largest series reports a success rate of $81 \%^{54}$ (Table 2). Enthusiasm for POEM is understandable in the setting of failed $\mathrm{HM}$, but the reported follow-up has generally been less than 1 year.

\section{Redo esophageal myotomy}

Reoperative myotomy with fundoplication is an option in cases of recurrence or persistence of symptoms owing to incomplete myotomy or myotomy scarring, with very good results reported (Table 3). ${ }^{29,31,55-63}$ Adhesions, fibrosis, and the loss of tissue planes in the area of the GEJ can make redo HM more difficult than the original operation, but laparotomy or laparoscopic approaches offer good visualization of the structures and surgeon experience can make a difference. ${ }^{28,31}$

Under direct view, and by sectioning the diaphragm in the midline from the hiatus, the esophagus can be gently separated from the liver, 
avoiding perforations. ${ }^{64}$ In an anterior wrap performed during the initial operation, the wrap should be mobilized to expose the anterior esophagus; however, if the fundoplication is a posteriorpositioned partial wrap and is intact, it can be preserved. ${ }^{31,61}$

The insertion of a 52-French bougie or the endoscope into the esophagus is useful in widening the organ, assisting in identifying the esophagus, and facilitating dissection. The myotomy should be extended at least $3-4 \mathrm{~cm}$ below the GEJ into the stomach and $6 \mathrm{~cm}$ proximally, along the esophagus. ${ }^{65,66}$ In the event of mucosal perforation, it is first closed, and then covered with a new fundoplication or extension of the preexisting fundoplication for coverage of the repair.

Often, redo myotomy is pursued only after failures of previous PD attempts, but many choose to redo the myotomy as the first line of intervention when faced with recurrence or persistence of dysphagia after HM. ${ }^{67,68}$ The most frequent complication reported is mucosal perforation, ${ }^{69}$ but there is no confirmation in the literature that the previous PD can increase the risk of perforation in the reoperation. ${ }^{70}$

\section{Esophagectomy}

Although end-stage achalasia occurs in less than $5 \%$ of all achalasia patients, persistent symptoms after failed dilation and/or myotomy may be disabling in up to $20 \%$ of these individuals, with dysphagia, regurgitation, nutritional failure, and respiratory complications being the most common complaints. ${ }^{58}$ Type III achalasia, inadequate or healed myotomy, and the development of peptic stricture, paraesophageal hernia, or carcinoma may be the cause of recurrent dysphagia and regurgitation after initial treatment of achalasia. ${ }^{71,72}$

In the case of recurrent symptoms with a massively dilated and tortuous esophagus, patients who had a previous pneumatic dilatation should be offered surgical myotomy. Patients with a failed myotomy should be offered a redo myotomy, and in the case of a new failure, esophagectomy. ${ }^{73}$ Owing to the associated morbidity and mortality risks, surgeons have long been reluctant to perform esophagectomy for a "benign" condition such as refractory achalasia.
Surgical myotomy and POEM are both effective therapies in patients with early-stage achalasia to reduce the pressure gradient across LES and to improve esophageal emptying. ${ }^{73-75}$ When patients have recurrent or persistent symptoms after esophageal myotomy, and revision therapy or redo myotomy fails to alleviate the symptoms, patients may be candidates for esophagectomy. In the absence of therapy or when therapy is ineffective, progressive dilatation and increasing tortuosity of the esophagus occur and result in worsening of outlet obstruction and retention of saliva and food. ${ }^{33}$ Esophageal resection may be required in patients with end-stage achalasia to restore alimentary transit, reverse nutritional deficiencies, and reduce the risk of aspiration pneumonia and carcinoma. End-stage achalasia is typically characterized by a dilated $(>6 \mathrm{~cm})$ and sigmoid esophagus (dolichomegaesophagus) and is diagnosed in about $5 \%$ of patients. ${ }^{75}$

Long-lasting achalasia is a well-known risk factor for esophageal squamous cell carcinoma (SSC). ${ }^{71}$ A study in esophagectomy specimens from patients with end-stage achalasia reported marked squamous hyperplasia, increased frequency of p53 immunoreactivity, and an increased number of $\mathrm{CD}^{+}$cells compared with controls. ${ }^{76}$ It has also been speculated that dilatation or myotomy could favor carcinogenesis by promoting esophageal reflux, Barrett's esophagus, and adenocarcinoma (AC). ${ }^{77}$ A meta-analysis showed that studies with a short-term follow-up can underestimate the risk of malignancy. In achalasia patients, the incidence and prevalence of SSC is 312 and 28 cases per 100,000 patients/year, respectively; moreover, the absolute risk increase for SCC is 308 and for AC is 183 cases per 100,000 patients/year. ${ }^{78}$

Management of end-stage achalasia remains controversial and although laparoscopic stapled cardioplasty has been recently revisited as a rescue procedure in selected patients, ${ }^{79-81}$ esophagectomy remains the standard therapy ${ }^{33,82-99}$ (Table 4).

A recent systematic review and meta-analysis ${ }^{99}$ of eight papers published between 1989 and 2014 and including 1307 patients showed that the mean age of the patient population was 55 ; half of the patients were females (50.5\%), and the average duration of symptoms before esophagectomy was $0.3-21$ years. Most patients had previous multiple endoscopic and/or surgical treatments and the indications for 
Table 4. Demographic and clinical data of patients undergoing esophagectomy for end-stage achalasia

\begin{tabular}{|c|c|c|c|c|c|c|}
\hline Author, year & Patients $(n)$ & Surgical approach $(n)$ & Replacement conduit $(n)$ & Morbidity (\%) & Mortality (\%) & $\begin{array}{l}\text { Follow-up } \\
\text { (months) }\end{array}$ \\
\hline Orringer and Stirling ${ }^{82}$ & 26 & TH (24) and TT (2) & Stomach & 19 & 0 & 30 \\
\hline Pinotti et al. ${ }^{83}$ & 122 & $\mathrm{TH}$ & Stomach & 19 & 4.1 & 60 \\
\hline Peters et al. ${ }^{84}$ & 19 & TT & Colon & 21 & 0 & 72 \\
\hline Miller et al. ${ }^{85}$ & 37 & TT (28) and TH (9) & $\begin{array}{l}\text { Stomach (31) } \\
\text { Colon (6) }\end{array}$ & 32.4 & 5.4 & 75 \\
\hline Banbury et al. ${ }^{86}$ & 32 & TH (21) and TT (11) & Stomach & 69 & 3 & 42 \\
\hline Devaney et al. ${ }^{87}$ & 93 & TH (87) and TT (6) & $\begin{array}{l}\text { Stomach (91) } \\
\text { Colon (2) }\end{array}$ & 30 & 2 & 38 \\
\hline Hsu et al. ${ }^{88}$ & 9 & LTA & Colon & 22 & 0 & 72 \\
\hline Gockel et al. ${ }^{89}$ & 8 & TH (6) and TT (2) & $\begin{array}{l}\text { Stomach (6) } \\
\text { Colon (2) }\end{array}$ & 25 & 0 & 44 \\
\hline Glatz et al. ${ }^{90}$ & 8 & TT & Stomach & 0 & 0 & 72 \\
\hline Palanivelu et al. ${ }^{91}$ & 11 & MIE-TH & Stomach & NR & 9 & 18 \\
\hline Tank et al..$^{92}$ & 15 & $\mathrm{TH}$ & Stomach & 50 & 5 & 12 \\
\hline Lewandowski ${ }^{93}$ & 7 & $\mathrm{TH}$ & $\begin{array}{l}\text { Jejunum (6) } \\
\text { Colon (1) }\end{array}$ & 0 & 0 & NR \\
\hline Schuchert et al..$^{94}$ & 6 & MIE-TT & Stomach & 50 & 0 & 24 \\
\hline Crema et al. ${ }^{95}$ & 60 & MIE-TH & Stomach & 20 & 0 & $6-118$ \\
\hline Howard et al. ${ }^{96}$ & 2 & $\mathrm{TH}$ & Stomach & 0 & 0 & 10.5 \\
\hline Carter et al..$^{97}$ & 4 & $\mathrm{TH}$ & Stomach & 50 & 0 & NR \\
\hline Molena et al. ${ }^{98}$ & 963 & TT & $\begin{array}{l}\text { Stomach (929) } \\
\text { Colon (34) }\end{array}$ & NR & 2.7 & NR \\
\hline Felix, $2016^{33}$ & 11 & MIE-TH & Stomach & 0 & 0 & $6-118$ \\
\hline Aiolfi et al. ${ }^{99}$ & 6 & $\begin{array}{c}\text { MIE-TT (5) and } \\
\text { MIE-TH (1) }\end{array}$ & Stomach & 33.3 & 0 & 70 \\
\hline
\end{tabular}

TH, transhiatal; TT, transthoracic; LTA, left thoracoabdominal; MIE, minimally invasive esophagectomy; NR, not reported.

esophagectomy were recurrent and disabling symptoms; in three patients, the resection was necessary because of the finding of SSC, and in the other two patients, a small focus of carcinoma was incidentally detected in the resected specimen. Esophagectomy was performed through a transthoracic $(78.7 \%)$ or a transhiatal (21.3\%) approach. In $95.3 \%$ of patients, the stomach was used as an esophageal substitute. Overall morbidity ranged from $19 \%$ to $50 \%$. The pooled prevalence of pneumonia was $10 \%(95 \%$ CI: 4-18\%), anastomotic leak 7\% (95\% CI: 4-10\%), and mortality $2 \%$ (95\% CI: $1-3 \%$ ). The reoperation rate was similar among patients who underwent the transhiatal and the transthoracic approach. Symptom resolution or improvement, unrestricted diet, and weight regain were recorded in most patients over a mean follow-up of 43 months. Only five series, including 195 patients, examined the longterm follow-up ( $>5$ years) after reconstruction with gastric or colon conduits, and the outcomes were similar in terms of symptomatic relief and quality of life.

A 10-year retrospective analysis of the Nationwide Inpatient Sample database analyzed outcomes following esophagectomy for achalasia between
2000 and $2010 .{ }^{98}$ Patients undergoing esophagectomy for achalasia $(n=963)$ were compared with a cohort undergoing esophagectomy for cancer $(n=18,003)$. A propensity-matched analysis showed a trend toward lower postoperative mortality in patients with achalasia $(2.9 \%$ versus $7.8 \%$, $P=0.08)$, although in-hospital mortality and complications were similar.

Esophagectomy for a benign clinical condition, such as refractory achalasia, is considered after all other treatment options have been exhausted and the symptoms of achalasia are considered refractory. The recent published UEG guidelines recommend that esophagectomy be considered only after all other treatments have been considered. ${ }^{38}$ Since the risk of SSC is much higher than in uncomplicated achalasia, patients with a longer than 10-year history of symptoms and multiple previous failures of myotomy and/or dilatation require regularly scheduled endoscopic surveillance. Esophagectomy should be performed earlier and without hesitation in patients who are fit for major surgery and present with disabling symptoms and dolichomegaesophagus recalcitrant to multiple endoscopic and laparoscopic interventions. With 
advances in techniques and available technologies, esophagectomy is safe and effective if performed in tertiary referral centers. It requires proper anesthesia management because the esophagus may be markedly dilated and filled with food debris, exposing the patient to the risk of aspiration pneumonia and respiratory failure. Awake intubation or rapid-sequence induction, and intubation in the semiupright position should be considered to prevent aspiration. A minimally invasive transhiatal or thoracoscopic approach is a feasible and safe alternative to the open surgical approach and may reduce respiratory complications. Compared with the transhiatal route, thoracoscopic mobilization provides improved visualization and magnification of the operative field, and easier dissection and hemostasis. The choice of the replacement conduit should be left to the surgeon's experience.

\section{Final comments}

In summary, management of achalasia remains challenging, and treatment of recurrent disease is not just challenging but potentially dangerous. When initial intervention for achalasia fails, regardless of the approach, each of the several treatment options-dilatation (pneumatic or standard), botulinum toxin, POEM, and revisional surgerycan be difficult and dangerous. Careful decision making about the method of second intervention is important and starting with the least invasive option is often prudent. The use of esophagectomy should be reserved for secondary treatment failures or when the esophagus has become so dilated that it is the only option for a chance of success. Finally, achalasia remains relatively uncommon and intervention should be undertaken by experienced providers or under their supervision since initial intervention is the safest and has the greatest chance for success.

\section{Competing interests}

The authors declare no competing interests.

\section{References}

1. Pandolfino, J.E. \& A.J. Gawron. 2015. Achalasia: a systematic review. JAMA 313: 1841-1852.

2. Allaix, M.E. \& M.G. Patti. 2013. What is the best primary therapy for achalasia: medical or surgical treatment? Who owns achalasia? J. Gastrointest. Surg. 17: 1547-1549.

3. Moonen, A., V. Annese, A. Belmans, et al. 2016. Long-term results of the European Achalasia Trial: a multicenter ran- domized controlled trial comparing pneumatic dilation versus laparoscopic Heller myotomy. Gut 65: 732-739.

4. Vaezi, M.F., J.E. Pandolfino \& M.F. Vela. 2013. ACG clinical guideline: diagnosis and management of achalasia. Am. J. Gastroenterol. 108: 1238-1249.

5. Leyden, J.E., A.C. Moss \& P. MacMathuna. 2014. Endoscopic pneumatic dilation versus botulinum toxin injection in the management of primary achalasia. Cochrane Database Syst. Rev. 12: 1-23.

6. Kadakia, S.C. \& R.K.H. Wong. 2001. Pneushmatic balloon dilation for esophageal achalasia. Gastrointest. Endosc. Clin. N. Am. 11: 325-345.

7. Felix, V.N. 2018. Results of pneumatic dilation in treating achalasia: predictive factors. Ann. N.Y. Acad. Sci. 1434: 124131.

8. Khashab, M.A., M.F. Vela, N. Thosani, et al. 2020. ASGE guideline on the management of achalasia. Gastrointest. Endosc. 91: 213-227.

9. Salvador, R., L. Spadotto, G. Capvilla, et al. 2016. Mucosal perforation during laparoscopic Heller myotomy has no influence on final treatment outcome. J. Gastrointest. Surg. 20: $1923-1930$.

10. Boeckxstaens, G.E., V. Annese, S.B. des Varannes, et al.; European Achalasia Trial Investigators. 2011. Pneumatic dilation versus laparoscopic Heller's myotomy for idiopathic achalasia. N. Engl. J. Med. 364: 1807-1816.

11. Litle, V.R. 2008. Laparoscopic Heller myotomy for achalasia: a review of the controversies. Ann. Thorac. Surg. 85: S743S746.

12. Torresan, F., A. Ioannou, F. Azzaroli \& F. Baszzoli. 2015. Treatment of achalasia in the era of high-resolution manometry. Ann. Gastroenterol. 28: 301-308.

13. Inoue, H., H. Minami, Y. Kobayashi, et al. 2010. Peroral endoscopic myotomy (POEM) for esophageal achalasia. Endoscopy 42: 265-271.

14. Crespin, O.M., L.W.C. Liu \& A. Parmar. 2017. et al. Safety and efficacy of POEM for treatment of achalasia: a systematic review of the literature. Surg. Endosc. 31: 21872201.

15. Ponds, F.A., P. Fockens, A. Lei, et al. 2019. Effect of peroral endoscopic myotomy vs pneumatic dilation on symptom severity and treatment outcomes among treatment-naive patients with achalasia: a randomized clinical trial. JAMA 322: 134-144.

16. Kim, G.H., K.W. Jung, H.Y. Jung, et al. 2019. Superior clinical outcomes of peroral endoscopic myotomy compared with balloon dilation in all achalasia subtypes. J. Gastroenterol. Hepatol. 34: 659-665.

17. Repici, A., L. Fuccio, R. Maselli, et al. 2018. GERD after per-oral endoscopic myotomy as compared with Heller's myotomy with fundoplication: a systematic review with meta-analysis. Gastrointest. Endosc. 87: 934-943.

18. Wang, H.M., W.C. Tai, S.K. Chuah, et al. 2015. Comparison of treatment outcomes of endoscope-guided pneumatic dilation and laparoscopic Heller myotomy. Kaohsiung J. Med. Sci. 31: 639-643.

19. Zaninotto, G., C. Bennet, G. Boeckxstaens, et al. 2018. The 2018 ISDE achalasia guidelines. Dis. Esophagus 31: 1-29.

20. Persson, J., E. Johnsson, S. Kostic, et al. 2015. Treatment of achalasia with laparoscopic myotomy or pneumatic 
dilatation: long-term results of a prospective, randomized study. World J. Surg. 39: 713-720.

21. Harvey, P.R., B. Coupland, J. Mytton, et al. 2019. Outcomes of pneumatic dilatation and Heller's myotomy for achalasia in England between 2005 and 2016. Gut 68: 1146-1151.

22. Werner, Y.B., B. Hakanson, J. Martinek, et al. 2019. Endoscopic or surgical myotomy in patients with idiopathic achalasia. N. Engl. J. Med. 381: 2219-2229.

23. Rawlings, A., N.J. Soper, B. Oelschlager, et al. 2012. Laparoscopic Dor versus Toupet fundoplication following Heller myotomy for achalasia: results of a multicenter, prospective, randomized-controlled trial. Surg. Endosc. 26: 18-26.

24. Csendes, A., I. Braghetto, P. Burdiles, et al. 2006. Very late results of esophagomyotomy for patients with achalasia: clinical, endoscopic, histologic, mano-metric, and acid reflux studies in 67 patients for a mean follow-up of 190 months. Ann. Surg. 243: 196-203.

25. Ortiz, A., L.F. de Haro, P. Parrilla, et al. 2008. Very long-term objective evaluation of Heller myotomy plus posterior partial fundoplication in patients with achalasia of the cardia. Ann. Surg. 247: 258-264.

26. Li, Q.L., L.Q. Yao, X.Y. Xu, et al. 2016. Repeat peroral endoscopic myotomy: a salvage option for persistent/recurrent symptoms. Endoscopy 48: 134-140.

27. Vaezi, M.F., M.E. Baker, E. Achkar, et al. 2002. Timed barium oesophagram: better predictor of long-term success after pneumatic dilation in achalasia than symptom assessment. Gut 50: 765-770.

28. Gockel, I., T. Junginger \& V.F. Eckardt. 2007. Persistent and recurrent achalasia after Heller myotomy: analysis of different patterns and long-term results of reoperation. Arch. Surg. 142: 1093-1097.

29. Patti, M.G. \& M.E. Allaix. 2015. Recurrent symptoms after Heller myotomy for achalasia: evaluation and treatment. World J. Surg. 39: 1625-1630.

30. Gockel, I., S. Timm, G.G. Sgourakis, et al. 2010. Achalasia - if surgical treatment fails: analysis of remedial surgery. J. Gastrointest. Surg. 14(Suppl. 1): S46-S57.

31. Serra, H.O., V.N. Felix, I. Cecconello, et al. 1998. Reapplication of myotomy and fundoplication in the surgical treatment of recurrent dysphagia after incomplete myotomy. Rev. Hosp. Clin. Fac. Med. São Paulo 53: 129-133.

32. Floch, N.R., R.A. Hinder, P.J. Klingler, et al. 1999. Is laparoscopic reoperation for failed antireflux surgery feasible? Arch. Surg. 134: 733-737.

33. Felix, V.N. 2016. Esophagectomy for end-stage achalasia. Ann. N.Y. Acad. Sci. 1381: 92-97.

34. Vela, M.F., J.E. Richter, D. Wachsberger, et al. 2004. Complexities of managing achalasia at a tertiary referral center: use of pneumatic dilatation, Heller myotomy, and botulinum toxin injection. Am. J. Gastroenterol. 99: 1029-1036.

35. Woodfield, C.A., M.S. Levine, S.E. Rubesin, et al. 2000. Diagnosis of primary versus secondary achalasia: reassessment of clinical and radiographic criteria. Am. J. Roentgenol. 175: 727-731.

36. Zaninotto, G., M. Costantini, G. Portale, et al. 2002. Etiology, diagnosis, and treatment of failures after laparoscopic Heller myotomy for achalasia. Ann. Surg. 235: 186-192.
37. Tatum, R.P., J.A. Wong, E.J. Figuerado, et al. 2007. Return of esophageal function after treatment for achalasia as determined by impedence-manometry. J. Gastrointest. Surg. 11: 1403-1409.

38. Oude Nijhuis, R.A.B., G. Zaninotto, S. Roman, et al. 2020. European guideline on achalasia - UEG and ESNM recommendations. United European Gastroenterol. J. 8: 13-34.

39. McW, W., S. AR, J.E. Richter, et al. 2020. Revisional procedures for recurrent symptoms after Heller myotomy and per-oral endoscopic myotomy. J. Lap. Adv. Surg. Tech. 30: 110-116.

40. Guardino, J.M., M.F. Vela, J.T. Connor, et al. 2004. Pneumatic dilation for the treatment of achalasia in untreated patients and patients with failed Heller myotomy. J. Clin. Gastroenterol. 38: 855-860.

41. Zaninotto, G., M. Costantini, C. Rizzetto, et al. 2008. Four hundred laparoscopic myotomies for esophageal achalasia: a single centre experience. Ann. Surg. 248: 986-993.

42. Chen, Z., J.R. Bessell, A. Chew, et al. 2010. Laparoscopic cardiomyotomy for achalasia: clinical outcomes beyond 5 years. J. Gastrointest. Surg. 14: 594-600.

43. Kumbhari, V., J. Behary, M. Szczesniak, et al. 2013. Efficacy and safety of pneumatic dilatation for achalasia in the treatment of post-myotomy symptom relapse. Am. J. Gastroenterol. 108: 1076-1081.

44. Legros, L., A. Ropert, C. Brochard, et al. 2014. Long-term results of pneumatic dilatation for relapsing symptoms of achalasia after Heller myotomy. Neurogastroenterol. Motil. 26: $1248-1255$.

45. Amani, M.F.N., S. Shirami, R. Malekzadeh, et al. 2015. Assessment of pneumatic balloon dilation in patients with symptomatic relapse after failed Heller myotomy. Middle East J. Dig. Dis. 8: 57-62.

46. Saleh, C.M., F.A. Ponds, M.P. Schijven, et al. 2016. Efficacy of pneumodilation in achalasia after failed Heller myotomy. Neurogastroenterol. Motil. 28: 1741-1746.

47. Schlottmann, F., C. Andolfi, R.T. Kavitt, et al. 2017. Multidisciplinary approach to esophageal achalasia: a single center experience. J. Lap. Adv. Surg. Tech. 27: 358-362.

48. Orenstein, S.B., S. Raigani, Y.V. Wu, et al. 2015. Peroral endoscopic myotomy (POEM) leads to similar results in patients with and without prior endoscopic or surgical therapy. Surg. Endosc. 29: 1064-1070.

49. Onimaru, M., H. Inoue, H. Ikeda, et al. 2013. Peroral endoscopic myotomy is a viable option for failed surgical esophagocardiomyotomy instead of redo surgical Heller myotomy: a single center prospective study. J. Am. Coll. Surg. 217: 598-605.

50. Zhou, P.H., Q.L. Li, L.Q. Yao, et al. 2013. Peroral endoscopic remyotomy for failed Heller myotomy: a prospective singlecenter study. Endoscopy 45: 161-166.

51. Vigneswaran, Y., A.K. Yetasook, J.C. Zhao, et al. 2014. Peroral endoscopic myotomy (POEM): feasible as reoperation following Heller myotomy. J. Gastrointest. Surg. 18: 10711076.

52. Fumagalli, U., R. Rosati, S. De Pascale, et al. 2016. Repeated surgical or endoscopic myotomy for recurrent dysphagia in patients after previous myotomy for achalasia. J. Gastrointest. Surg. 20: 494-499. 
53. Zhang, X., R.J. Modayil, D. Friedel, et al. 2018. Per-oral endoscopic myotomy in patients with or without prior Heller's myotomy: comparing long-term outcomes in a large U.S. single-center cohort (with videos). Gastrointest. Endosc. 87: 972-985

54. Ngamruengphong, S., H. Inoue, P.W. Chiu, et al. 2017. RETRACTED: long-term outcomes of per-oral endoscopic myotomy in patients with achalasia with a minimum followup of 2 years: an international multicenter study. Gastrointest. Endosc. 85: 927-933.

55. Rosati, R., U. Fumagalli, S. Bona, et al. 1998. Evaluating results of laparoscopic surgery for esophageal achalasia. Surg. Endosc. 12: 270-273.

56. Gorecki, P.J., R.A. Hinder, J.S. Libbey, et al. 2002. Redo laparoscopic surgery for achalasia. Surg. Endosc. 16: 772776.

57. Duffy, P.E., Z.T. Awad \& C.J. Filipi. 2003. The laparoscopic reoperation of failed Heller myotomy. Surg. Endosc. 17: 1046-1049.

58. Iqbal, A., B. Tierney, M. Haider, et al. 2006. Laparoscopic re-operation for failed Heller myotomy. Dis. Esophagus 19: 193-199.

59. Grotenhuis, B.A., B.P. Wijnhoven, J.C. Myers, et al. 2007. Reoperation for dysphagia after cardiomyotomy for achalasia. Am. J. Surg. 194: 678-682.

60. Rakita, S., D. Villadolid, C. Kalipersad, et al. 2007. Outcomes promote reoperative Heller myotomy for symptoms of achalasia. Surg. Endosc. 21: 1709-1714.

61. Herbella, F.A.M., J.L.B. Aquino, S. Stefani-Nakano, et al. 2008. Treatment of achalasia: lessons learned with Chagas' disease. Dis. Esophagus 21: 461-467.

62. Veenstra, B.R., R.F. Goldberg, S.P. Bowers, et al. 2016. Revisional surgery after failed esophagogastric myotomy for achalasia: successful esophageal preservation. Surg. Endosc. 30: $1754-1761$.

63. Smith, K.E., A.R. Saad, J.P. Hanna, et al. 2020. Revisional surgery in patients with recurrent dysphagia after Heller myotomy. J. Gastrointest. Surg. 24: 991-999.

64. Pinotti, H.W., B. Zilberstein, W. Pollara, et al. 1981. Esophagectomy without thoracotomy. Surg. Gynecol. Obstet. 152: 344-346.

65. Oelschlager, B.K., L. Chang \& C.A. Pellegrini. 2003. Improved outcome after extended gastric myotomy for achalasia. Arch. Surg. 138: 490-495.

66. Petersen, R.P. \& C.A. Pellegrini. 2010. Revisional surgery after Heller myotomy for esophageal achalasia. Surg. Laparosc. Endosc. Percutan. Tech. 20: 321-325.

67. Pallati, P.K. \& S.K. Mittal. 2011. Operative interventions for failed Heller myotomy: a single institution experience. Am. Surg. 77: 330-336.

68. Li, J., J. Lieb, M. Gianos, et al. 2012. Reasons and prevalence of reoperations after esophagomyotomy for achalasia. Surg. Laparosc. Endosc. Percutan. Tech. 22: 392-395.

69. Loviscek, M.F., A.S. Wright, M.W. Hinojosa, et al. 2013. Recurrent dysphagia after Heller myotomy: is esophagectomy always the answer? J. Am. Coll. Surg. 216: 736-743.

70. James, D.R., S. Purkayastha, O. Aziz, et al. 2012. The feasibility, safety and outcomes of laparoscopic re-operation for achalasia. Minim. Invasive Ther. Allied Technol. 21: 161-167.
71. Watson, T.J. 2015. Esophagectomy for end-stage achalasia. World J. Surg. 39: 1634-1641.

72. Herbella, F.A. \& M.G. Patti. 2015. Laparoscopic Heller myotomy and fundoplication in patients with end-stage achalasia. World J. Surg. 39: 1631-1633.

73. Bonavina, L. 2006. Minimally invasive surgery for esophageal achalasia. World J. Gastroenterol. 12: 59215925.

74. Kohn, G.P. 2019. Peroral endoscopic myotomy for achalasia. A review. Ann. Laparosc. Endosc. Surg. 4: 89.

75. Asti, E., A. Sironi, A. Lovece, et al. 2017. Health-related quality of life after laparoscopic Heller myotomy and Dor fundoplication for achalasia. Surgery 161: 977-983.

76. Peracchia, A., A. Segalin, R. Bardini, et al. 1991. Esophageal carcinoma and achalasia: prevalence, incidence and results of treatment. Hepatogastroenterology 38: 514-516.

77. Lehman, M.B., S.B. Clark, A.H. Ormsby, et al. 2001. Squamous mucosal alterations in esophagectomy specimens from patients with end-stage achalasia. Am. J. Surg. Pathol. 25: $1413-1418$.

78. Zendehdel, K., O. Nyren, A. Edberg, et al. 2011. Risk of esophageal adenocarcinoma in achalasia patients, a retrospective cohort study in Sweden. Am. J. Gastroenterol. 106: 57-61.

79. Dehn, T.C.B., M. Slater, N.J. Trudgill, et al. 2012. Laparoscopic stapled cardioplasty for failed treatment of achalasia. Br. J. Surg. 99: 1242-1245.

80. Agarwal, A.K. \& A. Javed. 2013. Laparoscopic esophagogastroplasty: a minimally invasive alternative to esophagectomy in the surgical management of megaesophagus with axis deviation. Surg. Endosc. 27: 2238-2242.

81. Griffiths, E.A., P.G. Devitt, G.G. Jamieson, et al. 2013. Laparoscopic stapled cardioplasty for end-stage achalasia. J. Gastrointest. Surg. 17: 997-1001.

82. Orringer, M.B. \& M.C. Stirling. 1989. Esophageal resection for achalasia: indications and results. Ann. Thorac. Surg. 47: 340-345.

83. Pinotti, H.W., I. Cecconello, J.M. da Rocha, et al. 1991. Resection for achalasia of the esophagus. Hepatogastroenterology 38: 470-473.

84. Peters, J.H., W.K. Kauer, P.F. Crookes, et al. 1995. Esophageal resection with colon interposition for end-stage achalasia. Arch. Surg. 130: 632-636.

85. Miller, D.L., M.S. Allen, V.F. Trastek, et al. 1995. Esophageal resection for recurrent achalasia. Ann. Thorac. Surg. 60: 922-925.

86. Banbury, M.K., T.W. Rice, J.R. Goldblum, et al. 1999. Esophagectomy with gastric reconstruction for achalasia. J. Thorac. Cardiovasc. Surg. 117: 1077-1084.

87. Devaney, E.J., M.D. Iannettoni, M.B. Orringer, et al. 2001. Esophagectomy for achalasia: patient selection and clinical experience. Ann. Thorac. Surg. 72: 854-858.

88. Hsu, H.S., C.Y. Wang, C.C. Hsieh \& M.H. Huang. 2003. Short-segment colon interposition for end-stage achalasia. Ann. Thorac. Surg. 76: 1706-1710.

89. Gockel, I., W. Kneist, V.F. Eckardt, et al. 2004. Subtotal esophageal resection in motility disorders of the esophagus. Dig. Dis. 22: 396-401. 
90. Glatz, S.M. \& J.D. Richardson. 2007. Esophagectomy for end stage achalasia. J. Gastrointest. Surg. 11: 11341137.

91. Palanivelu, C., M. Rangarajan, P.A. Jategaonkar, et al. 2008. Laparoscopic transhiatal esophagectomy for 'sigmoid' megaesophagus following failed cardiomyotomy: experience of 11 patients. Dig. Dis. Sci. 53: 1513-1518.

92. Tank, A.K., A. Kuma, T.L. Babu, et al. 2009. Resectional surgery in achalasia cardia. Int. J. Surg. 7: 155158.

93. Lewandowski, A. 2009. Diagnostic criteria and surgical procedure for megaesophagus-a personal experience. Dis. Esophagus 22: 305-309.

94. Schuchert, M.J., J.D. Luketich, R.J. Landreneau, et al. 2009. Minimally invasive surgical treatment of sigmoidal esophagus in achalasia. J. Gastrointest. Surg. 13: 1029-1035.
95. Crema, E., L.B. Ribeiro, R.C. Sousa, et al. 2009. Laparoscopic transhiatal esophagectomy for the treatment of advanced megaesophagus. An analysis of 60 cases. Rev. Col. Bras. Cir. 36: 118-122.

96. Howard, J.M., L. Ryan, K.T. Lim, et al. 2011. Oesophagectomy in the management of end-stage achalasia - case reports and a review of the literature. Int. J. Surg. 9: 204-208.

97. Carter, Y.M., C.D. Bond, S. Benjamin, et al. 2013. Minimally invasive transhiatal esophagectomy after thoracotomy. Ann. Thorac. Surg. 95: e41-e43.

98. Molena, D., B. Mungo, M. Stem, et al. 2014. Outcomes of esophagectomy for esophageal achalasia in the United States. J. Gastrointest. Surg. 18: 310-317.

99. Aiolfi, A., E. Asti, G. Bonitta \& L. Bonavina. 2018. Esophagectomy for end-stage achalasia: systematic review and meta-analysis. World J. Surg. 42: 1469-1476. 